\title{
Understanding the Proton Tautomerism Mechanism in Organic Molecular Ferroelectrics: Insights from Quantum Crystallography
}

\author{
Parthapratim Munshi", Sanjay Dutta and Anil Kumar \\ Chemical and Biological Crystallography Laboratory, Department of Chemistry, School of Natural Science, Shiv Nadar University, \\ Tehsil Dadri, Uttar Pradesh- 201314, India \\ parthapratim.munshi@snu.edu.in
}

Tautomeric systems such as keto-enol and enamine-imine incorporating both donor and acceptor in the same molecule, can be utilised to design single-component molecular ferroelectrics. This type of prototropic system reinforce intra or intermolecular hydrogen bonds synergistically with the augmentation of the $\pi$-system delocalization, defined as RAHB. ${ }^{1}$ The ferroelectric systems developed utilizing the aforementioned phenomenon are defined as proton tautomerizarion type ferroelectrics and the corresponding mechanism is known as proton tautomerism mechanism (PTM). ${ }^{2}$ However, this mechanism was never probed from electron density distributions. Here, we report the charge density analysis of 2-(4-(trifluoromethyl)phenyl)-1H-phenanthro [9,10-d] imidazole (1), the single-component molecular ferroelectric material (Figure 1). ${ }^{3}$ For elucidating the PTM in this molecular crystal, we have performed multipole model ${ }^{4}$ based experimental charge density analysis using high-resolution X-ray diffraction data. Thereby, we studied the deformation of electron densities (ED), the bond paths along with the bond critical points and the electrostatic potential (ESP) map along the N-H...N hydrogen bonds and performed topological analysis of the electron densities for understanding the underlying mechanism behind the proton transfer. The analysis also highlights the amphoteric characteristic of the enamine-imine unit (Figure 1). The different degrees of polarization of the electron densities of the N-H group and the N-atom of the adjacent molecule clearly supports the asymmetric N-

$\mathrm{H} . . . \mathrm{N}$ hydrogen bond characteristic in this displacive type ferroelectric. Such accurate analysis of multipole based electron densities further strengthen the understanding of the proton tautomerism effect in hydrogen bonded molecular ferroelectrics. To the best of our knowledge, this is the first report of charge density analysis on a molecular ferroelectric material. Our study points to the necessity of charge density analysis for improved understanding in this niche area of molecular ferroelectric research.
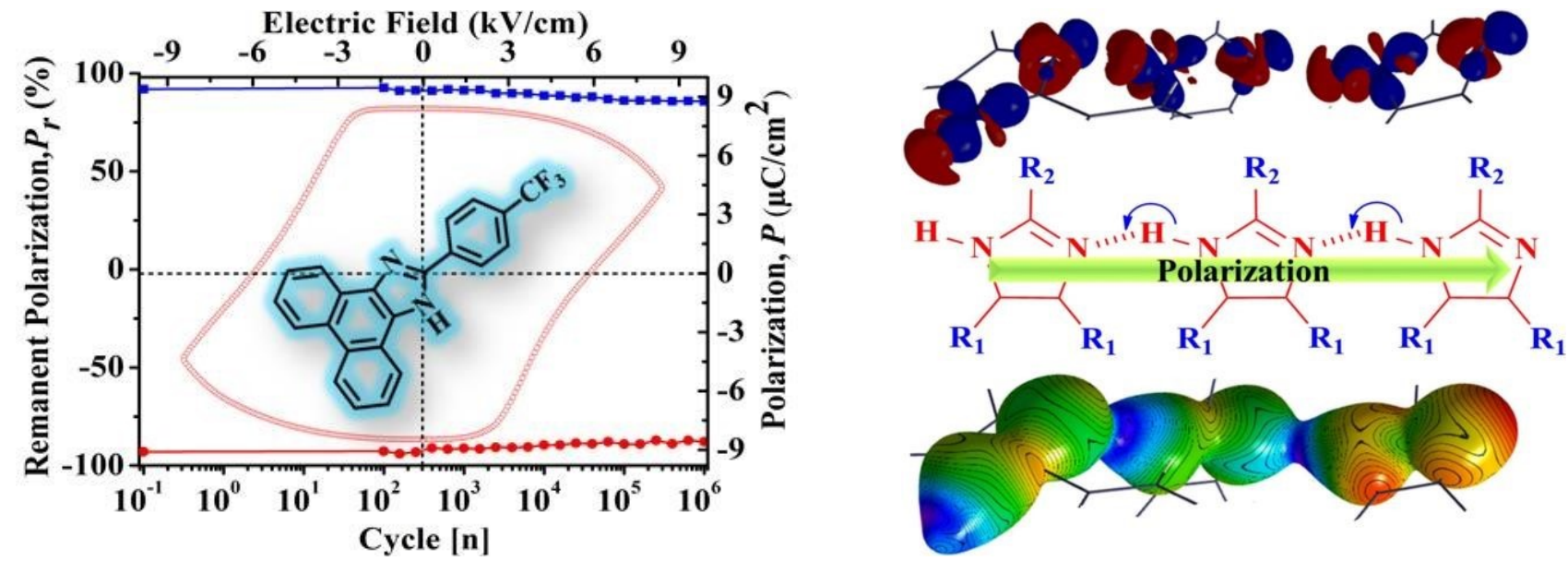

Figure 1: (left) $P-E$ loop and remanent polarization and (right) PTM pathway highlighting in terms of ED and ESP in 1

[1] P. Gilli, V. Bertolasi, V. Ferretti, G. Gilli, J. Am. Chem. Soc. 1994, 116 (3), 909-915.

[2] (a) S. Horiuchi, K. Kobayashi, R. Kumai, S. Ishibashi, Nat. Commun. 2017, 8, 1-9. (b) S. Dutta, V. Vikas, A. Yadav, R. Boomishankar, A. Bala, V. Kumar, T. Chakraborty, S. Elizabeth and P. Munshi, Chem. Commun., 2019, 55, 9610-9613.

[3] S. Dutta, Vikas, T. Vijayakanth and P. Munshi, Ferroelectric and Negative Thermal Expansion Properties in a purely organic multifunctional material. 2021 (to be published).

[4] N. K. Hansen and P. Coppens, Acta Crystallogr. Sect. A 1978, 34 (6), 909-921.

Keywords: Polarization; Proton Tautomerism; Molecular Ferroelectrics; Electron density; Quantum crystallography 